\title{
Effects of Hochuekkito combined with pulmonary rehabilitation in patients with chronic obstructive pulmonary disease
}

\author{
HIRONOBU HAMADA ${ }^{1,2}$, KIYOKAZU SEKIKAWA ${ }^{1}$, ISAO MURAKAMI ${ }^{3}$, \\ KOUICHI AIMOTO ${ }^{3}$, KAZUYOSHI KAGAWA ${ }^{4}$, TATSUYA SUMIGAWA ${ }^{4}$, KEN OKUSAKI $^{5}$, \\ TAKEFUMI DODO ${ }^{5}$, YOSHIKAZU AWAYA ${ }^{6}$, MASATOSHI WATANABE ${ }^{6}$, KEIICHI KONDO ${ }^{7}$, \\ TAKASHI OGAWA ${ }^{3}$, HIKARU YAMAMOTO ${ }^{1}$ and NOBORU HATTORI ${ }^{2}$
}

\begin{abstract}
Departments of ${ }^{1}$ Physical Analysis and Therapeutic Sciences, and ${ }^{2}$ Molecular and Internal Medicine, Graduate School of Biomedical and Health Sciences, Hiroshima University, Hiroshima, Hiroshima 734-8551; ${ }^{3}$ Department of Respiratory Medicine, Higashi-Hiroshima Medical Center, Higashi-Hiroshima, Hiroshima 739-0041; ${ }^{4}$ Department of Respiratory Medicine, Hiroshima City Medical Association-Administered Hiroshima City Aki Hospital, Hiroshima, Hiroshima 736-0088; ${ }^{5}$ Department of Internal Medicine, Mihara Medical Associations Hospital, Mihara, Hiroshima 723-0051; ${ }^{6}$ Department of Respiratory Medicine, Miyoshi Central Hospital, Miyoshi, Hiroshima 728-8502; ${ }^{7}$ Department of Respiratory Medicine, Tadanoumi Hospital, Takehara, Hiroshima 729-2316, Japan
\end{abstract}

Received May 13, 2018; Accepted October 2, 2018

DOI: $10.3892 /$ etm.2018.6837

\begin{abstract}
Chronic obstructive pulmonary disease (COPD) has significant systemic effects, such as weight loss, which affects exercise capacity, health-related quality of life (HRQOL) and survival. The traditional herbal medicine, Hochuekkito (TJ-41), improves the nutritional status and decreases systemic inflammation in patients with COPD. However, to date, the additive effect of TJ-41 on pulmonary rehabilitation (PR) in patients with COPD has not been researched comprehensively. The purpose of the present study was to investigate the efficacy and safety of adding TJ-41 to PR for patients with COPD. Thirty-three malnourished patients with COPD were randomly assigned to receive low-intensity exercise with (TJ-41 group) or without (control group) TJ-41 treatment for 12 weeks. The primary
\end{abstract}

Correspondence to: Professor Hironobu Hamada, Department of Physical Analysis and Therapeutic Sciences, Graduate School of Biomedical and Health Sciences, Hiroshima University, 1-2-3 Kasumi, Minami, Hiroshima 734-8551, Japan

E-mail: hirohamada@hiroshima-u.ac.jp

Abbreviations: 6MWD, 6-min walk distance; BZYQT, Bu-Zhong-Yi-Qi-Tang; CAT, chronic obstructive pulmonary disease assessment test; COPD, chronic obstructive pulmonary disease; FEV1, forced expiratory volume in $1 \mathrm{sec}$; FFM, fat-free mass; FVC, forced vital capacity; GOLD, Global Initiative for Chronic Obstructive Lung Disease; HPLC, high-performance liquid chromatography; HRQOL, health-related quality of life; IBW, ideal body weight; mMRC, modified Medical Research Council; PR, pulmonary rehabilitation; VAS, visual analog scale

Key words: chronic obstructive pulmonary disease, dyspnea, health-related quality of life, Hochuekkito, herbal medicine outcome was the change in the 6-min walk distance (6MWD). Secondary outcomes included changes in the body composition, peripheral muscle strength, modified Medical Research Council dyspnea score, visual analog scale (VAS) score for dyspnea, VAS score for fatigue and COPD assessment test (CAT) score. After the 12-week treatment, body weight and percent ideal body weight were significantly increased in the TJ-41 group $(\mathrm{P}<0.05)$, but not in the control group. After the 12-week treatment, the modified Medical Research Council dyspnea score, VAS score for dyspnea, VAS score for fatigue and total CAT score decreased significantly in the TJ-41 group (all $\mathrm{P}<0.05$ ), but not in the control group. There were no significant differences in the 6MWD and peripheral muscle strength between baseline and after 12 weeks of treatment in either group. No adverse effects were noted with the use of TJ-41. It was concluded that the addition of TJ-41 to PR may benefit malnourished patients with COPD with respect to dyspnea and HRQOL.

\section{Introduction}

Chronic obstructive pulmonary disease (COPD) is a progressive disease characterized by reduced expiratory airflow and dyspnea on exertion. Gradually progressing breathlessness limits daily activities and is a major reason for medical treatment in patients with COPD. COPD has significant systemic effects, such as weight loss and nutritional abnormalities, which affect exercise capacity, health-related quality of life (HRQOL), and survival (1-3). Potential mechanisms of weight loss are energy imbalance, disuse atrophy, hypoxemia, systemic inflammation, and hormone insufficiency (3).

Pulmonary rehabilitation (PR) is a useful non-pharmacological therapy that improves exercise capacity, dyspnea, and HRQOL in patients with COPD (4). Recent studies report that the combination of low-intensity exercise and nutritional support (5-7), or the combination of exercise training and 
ghrelin administration (8) may benefit malnourished patients with COPD. However, poor responders to nutritional therapy were older, and had lower appetites and more systemic inflammation (9).

A Japanese herbal medicine, Hochuekkito (i.e., TJ-41), is commonly used to treat chronic diseases or weakness after an illness. It improves the HRQOL and immune status in elderly individuals who complain of general fatigue or appetite loss due to chronic wasting disease $(10,11)$. In animal models of lung injury and viral infection, TJ-41 suppressed the production of inflammatory cytokines (12-14). Tatsumi et al (15) also reported that the administration of TJ-41 improved nutritional status, decreased the level of cytokines, and decreased the number of acute exacerbations in patients with COPD. However, to date, the additive effect of TJ-41 on PR in patients with COPD has not been well researched.

We hypothesized that the addition of TJ-41 treatment to PR may benefit malnourished patients with COPD, with respect to their exercise capacity, dyspnea, and HRQOL. In the present study, we evaluated the efficacy and safety of TJ-41 in malnourished patients with COPD who received PR.

\section{Materials and methods}

Study design. The study was a 12-week, open-label, randomized, parallel-group comparative study of TJ-41 administration during PR. It was conducted at six clinical hospitals from September 2014 to March 2016. The study was conducted in accordance with the Declaration of Helsinki guidelines and was approved by the Ethics Committees of Hiroshima University Hospital (Hiroshima, Japan), Higashi-Hiroshima Medical Center (Higashi-Hiroshima, Japan), Hiroshima City Medical Association-administered Hiroshima City Aki Hospital (Hiroshima, Japan), Mihara Medical Associations Hospital (Mihara, Japan), Miyoshi Central Hospital (Miyoshi, Japan), and Tadanoumi Hospital (Takehara, Japan). All participants provided written informed consent. This study was registered in the University Hospital Medical Information Network of Japan (http://www.umin.ac.jp/ctr/; number, UMIN000015092).

Patients. The inclusion criteria for the current study were as follows: i) moderate to severe COPD; defined by forced expiratory volume in 1 sec/forced vital capacity (FEV1/FVC) $<70 \%$ and FEV1\% predicted to be $>30 \%$ and $<80 \%$ [i.e., stages II and III, based on the classification of the Global Initiative for Chronic Obstructive Lung Disease (GOLD)] (16); ii) predicted percent ideal body weight (\%IBW) less than $100 \%$; iii) clinically stable disease and able to participate in PR for 12 weeks; iv) 40 years or older; and (5) smoking history of more than 10 pack-years.

Patients were excluded if they had any of the following: i) undergone PR within 24 weeks preceding the study; ii) a diagnosis of other pulmonary diseases or alpha1-antitrypsin deficiency; iii) diagnosis of an acute exacerbation within 4 weeks preceding the study; iv) received pulmonary transplantation; v) received herbal medicine for any problem within 4 weeks preceding the study; vi) had newly received bronchodilators or inhaled or systemic corticosteroids within 2 weeks before the study; vii) a diagnosis of other severe diseases, such as malignant tumors, autoimmune disease, liver disease, renal disease, heart disease, hematologic disease, and metabolic disease; viii) engaged in another clinical trial within 4 weeks preceding the study; ix) definitely or possibly pregnant; or $\mathrm{x}$ ) the physician judged the patient as unable to participate in the present study.

Study protocol. Randomization for all patients was accomplished manually by using a central registration system. Patients who met the eligibility criteria were enrolled and randomly assigned to receive PR with Group A [i.e., treated with TJ-41 powder (2.5 g; TJ-41, Extract Granules for Ethical Use, Tsumura Co., Tokyo Japan), which was taken orally three times per day before each meal or between meals] or Group B (i.e., untreated with TJ-41 powder). Randomization to Group A or Group B occurred at a 1:1 ratio, with stratification by the clinical stage of COPD. Within 4 weeks after enrollment and randomization, visits to the study institutes were scheduled on the first day of the administration of the study drug and PR, and every 2 weeks thereafter, throughout the 12-week treatment period.

Throughout the study period, patients were permitted to use a procaterol or salbutamol metered-dose inhaler, as needed, as well as stable doses of theophylline, long-acting $\beta_{2}$-agonist, long-acting muscarinic antagonist, and inhaled and oral corticosteroids. To control acute COPD exacerbations, investigators could administer any additional medication, such as antibiotics and systemic corticosteroids, as necessary.

In the present study, PR involved a home-based low-intensity exercise, as previously reported (6,7). Exercise training included upper and lower limb exercises, respiratory muscle stretching calisthenics, and level walking for at least $20 \mathrm{~min}$. Breathing retraining consisted of pursed-lip breathing, diaphragmatic breathing, and slow deep breathing in the supine and sitting positions. Patients were strongly instructed to practice this program daily at home and were supervised by respiratory therapists every 2 weeks. Relaxation and stair climbing exercises were also performed under the supervision of the respiratory therapists. The completion rate of PR was also checked when the patients visited the hospital.

Test drug. TJ-41 was obtained from the Ibaraki Plant of Tsumura and Co. (Tokyo, Japan). TJ-41 contains spray-dried hot water extracts of a mixture of the following medicinal plants: Astragali radix (16.7\%), Atractylodis lanceae rhizome $(16.7 \%)$, Ginseng radix (16.7\%), Angelicase radix $(12.5 \%)$, Bupleuri radix (8.3\%), Zizyphi fructus (8.3\%), Aurantii nobilis pericarpium (8.3\%), Glycyrrhizae radix (6.3\%), Cimicifugae rhizome (4.2\%), and Zingiberis rhizoma (2.0\%).

Outcome measurements. The primary outcome was a change in the 6-min walk distance (6MWD). Secondary outcomes were a change in body weight, \%IBW, body mass index (BMI), fat-free mass (FFM), pulmonary function, peripheral muscle strength, blood tests, modified Medical Research Council (mMRC) dyspnea scale score, visual analog scale (VAS) score for dyspnea, VAS score for fatigue, COPD assessment test (CAT) score, and the number of acute exacerbations.

The 6MWD was measured based on the ATS/ERS criteria $(17,18)$. Body height was measured to the nearest $0.5 \mathrm{~cm}$, and weight was measured to the nearest $0.1 \mathrm{~kg}$, using a weight and body composition meter (Omron, Kyoto, Japan). 
Table I. Baseline characteristics of patients with chronic obstructive pulmonary disease.

\begin{tabular}{lcc}
\hline Variables & Group A (n=18) & Group B (n=15) \\
\hline Age (years) & $75.3 \pm 6.1$ & $74.7 \pm 7.1$ \\
Sex & & \\
Male & $16(88.9)$ & $14(93.3)$ \\
Female & $2(11.1)$ & $1(6.7)$ \\
Height (cm) & $161.8 \pm 6.1$ & $159.0 \pm 7.9$ \\
Body weight $(\mathrm{kg})$ & $49.5 \pm 7.7$ & $50.0 \pm 4.8$ \\
Body mass index & $18.9 \pm 2.4$ & $19.8 \pm 1.4$ \\
GOLD stage & & \\
Stage II & $7(38.9)$ & $6(40.0)$ \\
Stage III & $11(61.1)$ & $9(60.0)$ \\
Medical treatment & & $12(80.0)$ \\
Use of LABA & $14(77.8)$ & $12(80.0)$ \\
Use of LAMA & $14(77.8)$ & $8(53.3)$ \\
Use of ICS & $7(38.9)$ & $1(6.7)$ \\
Use of theophylline & $2(11.1)$ &
\end{tabular}

The data are expressed as the number (percentage) for categorical variables and the mean \pm the standard deviation for numerical variables. GOLD, Global Initiative for Chronic Obstructive Lung Disease; ICS, inhaled corticosteroid; LABA, long-acting $\beta 2$-agonist; LAMA, long-acting muscarinic antagonist.

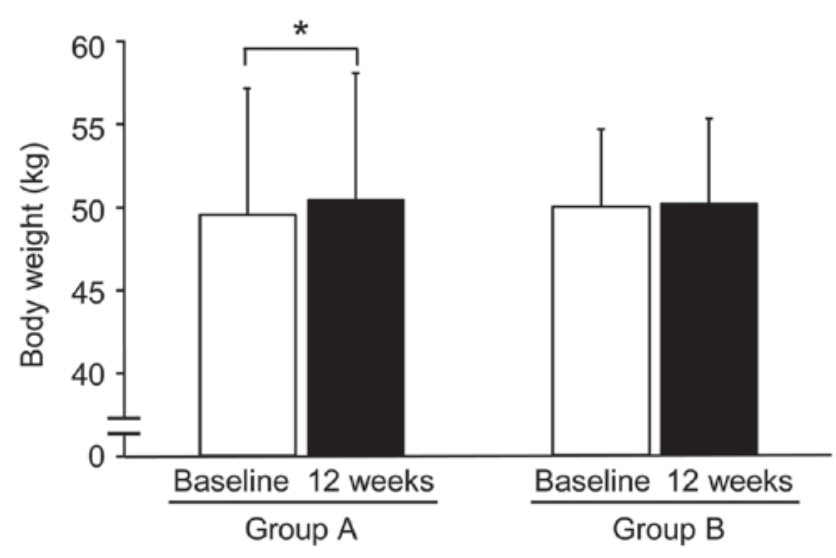

Figure 1. Changes in body weight after 12 weeks of treatment. Data are expressed as mean $\pm \mathrm{SD}$. ${ }^{*} \mathrm{P}<0.05$ vs. baseline.

The FFM was estimated using a bioelectrical impedance analysis. The BMI was calculated as the weight in kilograms divided by the square of the height value in meters. Pulmonary function parameters were measured based on the guidelines of ATS/ERS criteria $(16,19)$. For the muscle force parameters, peripheral muscle strength was measured by using the maximal voluntary handgrip maneuver. Dyspnea was assessed using the mMRC dyspnea scale, which is a questionnaire consisting of five statements about an individual's perceived breathlessness $(16,20)$. The severity of dyspnea in the patients were subjectively evaluated using the VAS, which consisted of a 10-cm horizontal line, indicating a score of 0 (i.e., no dyspnea) on the left end and 10 (i.e., extremely strong) on the right. In addition, the severity of fatigue was also assessed using a VAS. Each patient's HRQOL was evaluated using the CAT, which is a questionnaire used to assess the impact of COPD on the patient's health status $(21,22)$. The CAT has eight questions that assess cough, phlegm production, chest tightness, breathlessness, activity limitation, confidence, sleep, and energy. In blood tests, serum albumin level, hemoglobin level, white blood cell count, and lymphocyte counts (which are markers representing the nutritional or immunological status of patients) were measured. An acute exacerbation was defined as a complex of respiratory symptoms (i.e., new onset or an increase in at least one of the following symptoms: cough, sputum production, dyspnea, wheeze, and chest discomfort) lasting at least 3 days. These were generally treated with antibiotics and/or oral steroids $(16,23)$.

The 6MWD, pulmonary function, mMRC dyspnea scale score, and blood tests were measured at baseline and 12 weeks after treatment. Body composition parameters, peripheral muscle strength, VAS score for dyspnea, and VAS score for fatigue were measured at baseline, and subsequently, every 4 weeks.

Safety measurements. Adverse events were assessed throughout the $12^{-}$week study period. Physical examinations and blood tests, such as liver function tests (e.g., levels of alanine aminotransferase, aspartate aminotransferase, alkaline phosphatase, $\gamma$-glutamyl transpeptidase, and total bilirubin) and the potassium level were measured as markers representing adverse events. These markers were measured at baseline, and subsequently, every 4 weeks.

Statistical analysis. Results are presented as the mean \pm standard deviation. Partially missing data of the clinical evaluation were carried forward using the principle of the Last Observation Carried Forward. Data between the groups were compared using Fisher's exact tests and Mann-Whitney $U$ tests. Data at baseline and 12 weeks were compared using a Wilcoxon signed-ranks test. Statistical analyses were performed using IBM SPSS version 21 software for Windows (IBM Japan, Tokyo, Japan). Statistical significance was set at $\mathrm{P}<0.05$.

\section{Results}

Patients' characteristics. A total of 35 patients were randomized in the current study; 2 patients (1 patient at stage II and 1 patient at stage III) in Group B were excluded from the analysis because of withdrawal. Thus, 33 patients were finally enrolled in this study. A total of 18 patients ( 7 patients at stage II and 11 patients at stage III) received TJ-41 (i.e., Group A), while 15 patients (6 patients at stage II and 9 patients at stage III) did not receive TJ-41 (i.e., Group B).

At baseline, there were no significant differences in age, BMI, and medical treatment between the groups (Table I). In addition, no significant differences were observed in FFM, FEV1\% predicted, 6MWD, mMRC dyspnea score, VAS score for fatigue, total CAT score, and serum albumin and hemoglobin levels between the groups at baseline.

Outcomes. The body weight (Fig. 1) and \%IBW were significantly increased after 12 weeks of treatment in Group A, but not in Group B. There were no significant differences 
Table II. Changes in body composition, muscle force, health-related quality of life and blood tests.

\begin{tabular}{|c|c|c|c|c|}
\hline \multirow[b]{2}{*}{ Variable } & \multicolumn{2}{|c|}{ Group A $(n=18)$} & \multicolumn{2}{|c|}{ Group B $(n=15)$} \\
\hline & Baseline & 12 weeks & Baseline & 12 weeks \\
\hline Body weight (kg) & $49.5 \pm 7.7$ & $50.1 \pm 8.1^{\mathrm{b}}$ & $50.0 \pm 4.8$ & $50.1 \pm 5.3$ \\
\hline Body mass index & $18.9 \pm 2.4$ & $19.1 \pm 2.5$ & $19.8 \pm 1.4$ & $19.8 \pm 1.7$ \\
\hline Ideal body weight ( $\%$ predicted) & $85.0 \pm 10.7$ & $86.8 \pm 11.2^{\mathrm{b}}$ & $89.9 \pm 6.6$ & $90.2 \pm 7.7$ \\
\hline Fat free mass $(\mathrm{kg})$ & $38.9 \pm 5.2$ & $39.1 \pm 5.4$ & $38.5 \pm 5.3$ & $38.9 \pm 5.3$ \\
\hline FVC (1) & $2.3 \pm 0.8$ & $2.3 \pm 0.7$ & $2.6 \pm 0.7$ & $2.7 \pm 0.6$ \\
\hline FEV1 (l) & $1.1 \pm 0.5$ & $1.0 \pm 0.5$ & $1.1 \pm 0.5$ & $1.2 \pm 0.5^{\mathrm{b}}$ \\
\hline FEV1 (\% predicted) & $46.9 \pm 18.4$ & $44.9 \pm 18.6$ & $49.5 \pm 15.8$ & $51.3 \pm 16.2$ \\
\hline FEV1/FVC (\%) & $47.1 \pm 14.4$ & $44.3 \pm 11.2$ & $42.4 \pm 7.9$ & $44.4 \pm 9.0$ \\
\hline 6MWD (m) & $335.3 \pm 102.1$ & $341.8 \pm 103.8$ & $363.7 \pm 98.7$ & $359.0 \pm 125.1$ \\
\hline Handgrip strength (kg) & $28.4 \pm 6.5$ & $28.2 \pm 6.1$ & $27.7 \pm 8.0$ & $28.7 \pm 8.0$ \\
\hline mMRC dyspnea scale score & $2.1 \pm 1.0$ & $1.5 \pm 1.0^{\mathrm{b}}$ & $1.8 \pm 0.9$ & $1.6 \pm 0.6$ \\
\hline VAS score for dyspnea & $5.4 \pm 2.4^{\mathrm{a}}$ & $3.7 \pm 2.4^{\mathrm{b}}$ & $4.0 \pm 1.7$ & $4.3 \pm 3.1$ \\
\hline VAS score for fatigue & $4.8 \pm 2.4$ & $3.5 \pm 2.3^{\mathrm{b}}$ & $4.3 \pm 1.8$ & $4.4 \pm 2.3$ \\
\hline Total CAT score & $18.4 \pm 7.4$ & $12.2 \pm 8.1^{\mathrm{c}}$ & $17.8 \pm 9.4$ & $16.2 \pm 9.9$ \\
\hline Score of cough & $2.2 \pm 1.5$ & $1.1 \pm 1.5^{\mathrm{c}}$ & $1.4 \pm 1.5$ & $1.8 \pm 1.6$ \\
\hline Score of production of phlegm & $1.6 \pm 1.6$ & $1.0 \pm 1.4^{\mathrm{b}}$ & $2.1 \pm 1.9$ & $2.2 \pm 1.7$ \\
\hline Score of chest tightness & $2.6 \pm 1.5$ & $1.8 \pm 1.3^{\mathrm{b}}$ & $2.6 \pm 1.2$ & $2.1 \pm 1.5$ \\
\hline Score of breathlessness & $3.8 \pm 1.2$ & $2.9 \pm 1.3^{\mathrm{b}}$ & $3.6 \pm 1.3$ & $3.4 \pm 1.3$ \\
\hline Score of activity limitation & $1.6 \pm 1.5$ & $1.2 \pm 1.3$ & $1.9 \pm 1.7$ & $1.5 \pm 1.7$ \\
\hline Score of confidence & $1.8 \pm 1.7$ & $1.2 \pm 1.3^{\mathrm{b}}$ & $1.9 \pm 1.9$ & $1.4 \pm 1.7$ \\
\hline Score of sleep & $1.9 \pm 1.5$ & $1.0 \pm 1.2^{\mathrm{b}}$ & $1.7 \pm 1.5$ & $1.9 \pm 1.4$ \\
\hline Score of energy & $2.8 \pm 1.4$ & $1.9 \pm 1.4^{\mathrm{b}}$ & $2.6 \pm 1.5$ & $2.0 \pm 1.3$ \\
\hline Serum albumin (g/dl) & $4.3 \pm 0.2$ & $4.2 \pm 0.3$ & $4.1 \pm 0.3$ & $4.0 \pm 0.3$ \\
\hline Hemoglobin $(\mathrm{g} / \mathrm{dl})$ & $13.7 \pm 1.6$ & $13.6 \pm 1.8$ & $13.9 \pm 1.3$ & $13.8 \pm 1.1$ \\
\hline Lymphocyte counts $(/ \mu 1)$ & $1794.0 \pm 517.4$ & $1809.9 \pm 827.0$ & $1793.0 \pm 671.0$ & $1724.6 \pm 716.0$ \\
\hline
\end{tabular}

The data are expressed as the mean \pm the standard deviation. ${ }^{a} \mathrm{P}<0.05$ vs. group $\mathrm{B}$. ${ }^{\mathrm{b}} \mathrm{P}<0.05,{ }^{\mathrm{c}} \mathrm{P}<0.005$ vs. baseline. $6 \mathrm{MWD}, 6$-min walk distance; CAT, chronic obstructive pulmonary disease assessment test; FEV1, forced expiratory volume in 1 second; FVC, forced vital capacity; mMRC, modified Medical Research Council; ns, not significant; VAS, visual analog scale.

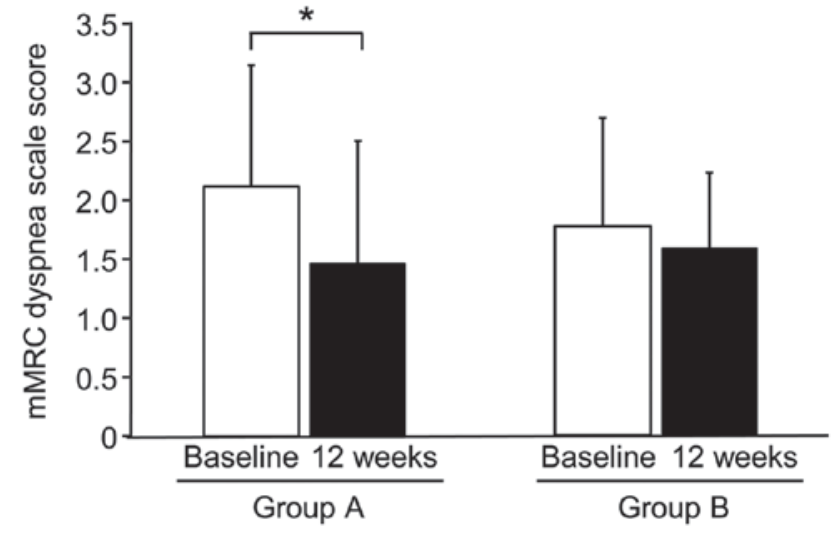

Figure 2. Changes in mMRC dyspnea scale score after 12 weeks of treatment. Data are expressed as mean \pm SD. ${ }^{*} \mathrm{P}<0.05$ vs. baseline. mMRC, modified Medical Research Council.

in either group in the other parameters of body composition, between baseline and after the 12 -week treatment. The mMRC dyspnea scores (Fig. 2), VAS scores for dyspnea,

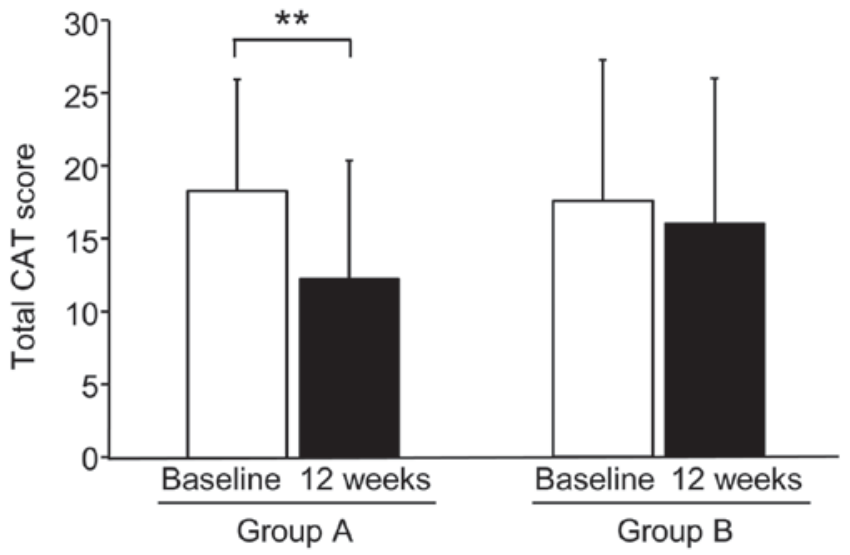

Figure 3. Changes in total CAT score after 12 weeks of treatment. Data are expressed as mean $\pm \mathrm{SD}$. ${ }^{* *} \mathrm{P}<0.005$ vs. baseline. CAT, COPD assessment test.

VAS scores for fatigue, and total CAT scores (Fig. 3) were significantly decreased after 12 weeks of treatment in Group A, but not in Group B. In particular, the CAT scores of 7 of 
8 individuals in Group A were significantly decreased after 12 weeks of treatment. No significant differences were noted in the 6MWD, peripheral muscle strength, and blood markers between baseline and 12 weeks after treatment in either group. The values of FEV1 in Group B were increased after 12 weeks of treatment; however, the predicted values of FEV1 were not increased. Table II shows changes in the parameters of body composition, pulmonary function, muscle force, respiratory symptoms, HRQOL, and blood markers.

Adverse Events. No patient experienced TJ-41 treatment-related adverse events, such as liver dysfunction and hypokalemia. One patient in Group A died due to dissection of an abdominal aneurysm. One patient in Group B developed pneumonia and required hospitalization. Two patients in Group B experienced acute exacerbations of COPD, but no patients in Group A had such an experience.

\section{Discussion}

In the present study, adding TJ-41 treatment to PR significantly increased a patient's body weight, and significantly decreased the mMRC dyspnea scores, VAS scores for dyspnea, VAS scores for fatigue, and total CAT scores of patients with COPD. TJ-41 combined with PR may benefit malnourished patients with COPD, with respect to dyspnea and HRQOL.

The addition of TJ-41 treatment to PR may have beneficial effects on dyspnea scores and HRQOL scores in malnourished COPD patients. A previous meta-analysis (24) demonstrated the efficacy of the Chinese herbal medicine, Bu-Zhong-Yi-Qi-Tang (BZYQT), in patients with stable COPD. Many studies have examined the utility of drugs similar to TJ-41 in this population, but the benefits of TJ-41 in patients with stable COPD remain largely unexplored. The investigators in two studies reported that BZYQT improved CAT scores (24). Tatsumi et al (15) reported that after 6 months of treatment, the symptoms score on the St. George's Respiratory Questionnaire decreased in patients with COPD receiving TJ-41, but did not decrease in the control group. This finding indicated that TJ-41 improved HRQOL in patients with COPD. Furthermore, the beneficial effects of TJ-41 on cancer-related fatigue and HRQOL in patients with cancer have also been reported (25). In the present study, the mMRC dyspnea scores, VAS scores for dyspnea, VAS scores for fatigue, and total CAT scores significantly decreased after 3 months of treatment in Group A, but not in Group B. The scores of individual CAT parameters, including sleep and pulmonary symptoms, significantly decreased in Group A.

TJ-41 improved systemic inflammation and nutritional status. It suppressed the production of inflammatory cytokines in animal models of lung injury and viral infection (12-14). In patients with COPD, Tatsumi et al (15) reported decreased serum levels of inflammatory proteins, such as C-reactive protein, tumor necrosis factor alpha, and interleukin-6, and increased serum levels of prealbumin, a nutritional marker, after 6 months of TJ-41 treatment; however, these levels remained unchanged in the control group. They also showed that body weight increased from baseline to the end of the trial in the TJ-41 group, but remained unchanged in the control group. Compared to the control group, patients with
Mycobacterium avium complex disease showed increased body weight and serum albumin levels when treated with TJ-41 (26). In the present study, body weight and \%IBW in Group A were significantly increased after 3 months of treatment, but not in Group B. However, there was no significant change in serum albumin levels in either group.

The reason for the improvement in the dyspnea scores and HRQOL scores after TJ-41 administration in patients with COPD is unclear. Improvements in systemic inflammation and the nutritional status may have ameliorated dyspnea scores and HRQOL scores in patients with COPD. Improvements in the scores of dyspnea, cough, and breathlessness could be attributed to the decrease of phlegm production, based on the anti-inflammatory effects of TJ-41. HRQOL seems to have consequently increased due to the improvement of these scores. In addition, Katsura et al (27) reported that low body weight in patients with COPD is associated with worsening dyspnea and a deterioration in generic and disease-specific HRQOL. Further, the antidepressive effect of TJ-41 may improve the dyspnea score and HRQOL score in patients with COPD. It was reported that large numbers of patients with COPD had clinically significant levels of depression and/or anxiety $(28,29)$. Tohda and Mingmalairak (30) reported that TJ-41 had an antidepressive effect in a mouse model of depression with learned helplessness behavior. Further study is needed to clarify the antidepressive effect of TJ-41 in patients with COPD.

Although several studies (24) demonstrated the efficacy of BZYQT on the 6MWD in stable patients with COPD, the addition of TJ-41 treatment to PR did not improve exercise capacity in patients with COPD in the current study. The reason for this discrepancy is unclear. In our study, TJ-41 increased body weight, but not FFM or muscle strength in patients with COPD, which may have contributed to our discrepant findings. It was reported that the reduction in FFM is a better predictor of exercise performance compared to the BMI $(31,32)$. In addition, patients with a low FFM often have muscular weakness, especially in the lower limbs $(33,34)$. Further study is needed to clarify the effects of TJ-41 on exercise capacity in patients with COPD.

Several limitations in the current study must be noted. First, it only included a small number of patients. We screened approximately double the number of enrolled malnourished patients with COPD, but many patients were excluded due to several reasons, such as taking herbal medicines, having a diagnosis of severe diseases or orthopedic diseases, and refusal to participate in the study. Second, our study was an open label study without the administration of a placebo. It was very difficult to produce a placebo for an herbal medicine that contains many active ingredients. In addition, this is the first pilot study to evaluate the effect of an herbal medicine combined with PR in patients with COPD. Thus, we designed it as an open-label, randomized, parallel-group comparative study. Larger randomized placebo-controlled studies are required to validate the findings of this study.

Our study demonstrated that the addition of TJ-41 treatment to PR improved body weight, dyspnea scores, and HRQOL scores in patients with COPD. No adverse events were observed after 12 weeks of TJ-41 treatment. TJ-41 combined with PR may be a new therapeutic strategy to treat patients with COPD. 


\section{Acknowledgements}

The abstract was presented at the European Respiratory Society International Congress 2017, September 9-13 2017 in Milan, and published as abstract no. PA3712 in European Respiratory Journal 50 (suppl 61), 2017.

\section{Funding}

The present study was supported by the Fundamental Budget for Education and Research and the pharmaceutical company Tsumura and Co., which provided the TJ-41.

\section{Availability of data and materials}

All data generated or analyzed during this study are included in this published article.

\section{Authors' contributions}

$\mathrm{HH}, \mathrm{KS}$ and NH designed the study and drafted the manuscript. $\mathrm{HH}$ and KS participated in the statistical design and analyses. $\mathrm{HH}, \mathrm{IM}, \mathrm{KaK}, \mathrm{KO}$, YA, KeK and TO recruited patients in the hospitals. KS, KA, TS, TD, MW and HY engaged in PR. All authors have read and approved the final manuscript.

\section{Ethics approval and consent to participate}

The study was approved by the ethics committees of Hiroshima University Hospital, Higashi-Hiroshima Medical Center, Hiroshima City Medical Association-administered Hiroshima City Aki Hospital, Mihara Medical Associations Hospital, Miyoshi Central Hospital, and Tadanoumi Hospital. All participants provided written informed consent.

\section{Patient consent for publication}

Not applicable.

\section{Competing interests}

The present study received partial financial support from the pharmaceutical company Tsumura and Co. However, the company had no control over the interpretation, writing, or publication of the present study. The authors have no competing interests directly relevant to the content of this article.

\section{References}

1. Schols A: Nutritional modulation as part of the integrated management of chronic obstructive pulmonary disease. Proc Nutr Soc 62: 783-791, 2003.

2. Cao C, Wang R, Wang J, Bunjhoo H, Xu Y and Xiong W: Body mass index and mortality in chronic obstructive pulmonary disease: A meta-analysis. PLoS One 7: e43892, 2012.

3. Wagner PD: Possible mechanisms underlying the development of cachexia in COPD. Eur Respir J 31: 492-501, 2008.

4. McCarthy B, Casey D, Devane D, Murphy K, Murphy E and Lacasse Y: Pulmonary rehabilitation for chronic obstructive pulmonary disease. Cochrane Database Syst Rev: CD003793, 2015.

5. Ferreira IM, Brooks D, White J and Goldstein R: Nutritional supplementation for stable chronic obstructive pulmonary disease. Cochrane Database Syst Rev 12: CD000998, 2012.
6. Sugawara K, Takahashi H, Kasai C, Kiyokawa N, Watanabe T, Fujii S, Kashiwagura T, Honma M, Satake M and Shioya T: Effects of nutritional supplementation combined with low-intensity exercise in malnourished patients with COPD. Respir Med 104: 1883-1889, 2010.

7. Sugawara K, Takahashi H, Kashiwagura T, Yamada K, Yanagida S, Homma M, Dairiki K, Sasaki H, Kawagoshi A, Satake M and Shioya T: Effect of anti-inflammatory supplementation with whey peptide and exercise therapy in patients with COPD. Respir Med 106: 1526-1534, 2012.

8. Miki K, Maekura R, Nagaya N, Nakazato M, Kimura H, Murakami S, Ohnishi S, Hiraga T, Miki M, Kitada S, et al: Ghrelin treatment of cachectic patients with chronic obstructive pulmonary disease: A multicenter, randomized, double-blind, placebo-controlled trial. PLoS One 7: e35708, 2012.

9. Creutzberg EC, Schols AM, Weling-Scheepers CA, Buurman WA and Wouters EF: Characterization of nonresponse to high caloric oral nutritional therapy in depleted patients with chronic obstructive pulmonary disease. Am J Respir Crit Care Med 161: 745-752, 2000.

10. Kuroiwa A, Liou S, Yan H, Eshita A, Naitoh S and Nagayama A: Effect of a traditional Japanese herbal medicine, hochu-ekki-to (Bu-Zhong-Yi-Qi Tang), on immunity in elderly persons. Int Immunopharmacol 4: 317-324, 2004.

11. Satoh N, Sakai S, Kogure T, Tahara E, Origasa H, Shimada Y, Kohoda K, Okubo T and Terasawa K: A randomized double blind placebo-controlled clinical trial of Hochuekkito, a traditional herbal medicine, in the treatment of elderly patients with weakness $\mathrm{N}$ of one and responder restricted design. Phytomedicine 12: 549-554, 2005.

12. Mori K, Kido T, Daikuhara H, Sakakibara I, Sakata T, Shimizu K, Amagaya S, Sasaki H and Komatsu Y: Effect of Hochu-ekki-to (TJ-41), a Japanese herbal medicine, on the survival of mice infected with influenza virus. Antiviral Res 44: 103-111, 1999.

13. Tajima S, Bando M, Yamasawa $\mathrm{H}$, Ohno S, Moriyama $\mathrm{H}$ Takada T, Suzuki E, Gejyo F and Sugiyama Y: Preventive effect of Hochu-ekki-to on lipopolysaccharide-induced acute lung injury in BALB/c mice. Lung 184: 318-323, 2006.

14. Tajima S, Bando M, Yamasawa H, Ohno S, Moriyama H, Terada M, Takada T, Suzuki E, Gejyo F and Sugiyama Y: Preventive effect of hochu-ekki-to, a Japanese herbal medicine, on bleomycin-induced lung injury in mice. Respirology 12: 814-822, 2007.

15. Tatsumi K, Shinozuka N, Nakayama K, Sekiya N, Kuriyama T and Fukuchi Y: Hochuekkito improves systemic inflammation and nutritional status in elderly patients with chronic obstructive pulmonary disease. J Am Geriatr Soc 57: 169-170, 2009.

16. Global initiative for chronic obstructive lung disease: Global strategy for the diagnosis, management, and prevention of chronic obstructive pulmonary disease. 2018 Report. http://www.goldcopd. org. Accessed on July 12, 2018.

17. ATS Committee on Proficiency Standards for Clinical Pulmonary Function Laboratories: ATS statement: Guidelines for the six-minute walk test. Am J Respir Crit Care Med 166: 111-117, 2002.

18. Holland AE, Spruit MA, Troosters T, Puhan MA, Pepin V, Saey D, McCormack MC, Carlin BW, Sciurba FC, Pitta F, et al: An official European Respiratory Society/American Thoracic Society technical standard: Field walking tests in chronic respiratory disease. Eur Respir J 44: 1428-1446, 2014.

19. Miller MR, Hankinson J, Brusasco V, Burgos F, Casaburi R, Coates A, Crapo R, Enright P, van der Grinten CP, Gustafsson P, et al: Standardisation of spirometry. Eur Respir J 26: 319-338, 2005.

20. Celli BR and MacNee W; ATS/ERS Task Force: Standards for the diagnosis and treatment of patients with COPD: A summary of the ATS/ERS position paper. Eur Respir J 23: 932-946, 2004.

21. Jones PW, Harding G, Berry P, Wiklund I, Chen WH and Kline Leidy N: Development and first validation of the COPD Assessment Test. Eur Respir J 34: 648-654, 2009.

22. Glaxo Smith Kline group of companies: COPD Asessment Test 2016. http://www.catestonline.org. Accessed on July 12, 2018.

23. Vogelmeier C, Hederer B, Glaab T, Schmidt H, Rutten-van Mölken MP, Beeh KM, Rabe KF and Fabbri LM; POET-COPD investigators: Tiotropium versus salmeterol for the prevention of exacerbations of COPD. N Engl J Med 364: 1093-1103, 2011.

24. Chen Y, Shergis JL, Wu L, Yu X, Zeng Q, Xu Y, Guo X, Zhang AL, Xue CC and Lin L: A systematic review and meta-analysis of the herbal formula Buzhong Yiqi Tang for stable chronic obstructive pulmonary disease. Complement Ther Med 29: 94-108, 2016. 
25. Jeong JS, Ryu BH, Kim JS, Park JW, Choi WC and Yoon SW: Bojungikki-tang for cancer-related fatigue: A pilot randomized clinical trial. Integr Cancer Ther 9: 331-338, 2010.

26. Enomoto Y, Hagiwara E, Komatsu S, Nishihira R, Baba T, Kitamura H, Sekine A, Nakazawa A and Ogura T: Pilot quasi-randomized controlled study of herbal medicine Hochuekkito as an adjunct to conventional treatment for progressed pulmonary Mycobacterium avium complex disease. PLoS One 9: e104411, 2014.

27. Katsura H, Yamada K and Kida K: Both generic and disease specific health-related quality of life are deteriorated in patients with underweight COPD. Respir Med 99: 624-630, 2005.

28. Kunik ME, Roundy K, Veazey C, Souchek J, Richardson P, Wray NP and Stanley MA: Surprisingly high prevalence of anxiety and depression in chronic breathing disorders. Chest 127: 1205-1211, 2005.

29. Hanania NA, Müllerova H, Locantore NW, Vestbo J, Watkins ML, Wouters EF, Rennard SI and Sharafkhaneh A; Evaluation of COPD Longitudinally to Identify Predictive Surrogate Endpoints (ECLIPSE) study investigators: Evaluation of COPD Longitudinally to Identify Predictive Surrogate Endpoints (ECLIPSE) study investigators. Determinants of depression in the ECLIPSE chronic obstructive pulmonary disease cohort. Am J Respir Crit Care Med 183: 604-611, 2011.

30. Tohda M and Mingmalairak S: Evidence of antidepressive effects of a Wakan-yaku, Hochuekkito, in depression model mice with learned-helplessness behavior. Evid Based Complement Alternat Med 2013: 319073, 2013
31. Baarends EM, Schols AM, Mostert R and Wouters EF: Peak exercise response in relation to tissue depletion in patients with chronic obstructive pulmonary disease. Eur Respir J 10: 2807-2813, 1997.

32. Sabino PG, Silva BM and Brunetto AF: Nutritional status is related to fat-free mass, exercise capacity and inspiratory strength in severe chronic obstructive pulmonary disease patients. Clinics (Sao Paulo) 65: 599-605, 2010.

33. Franssen FM, Broekhuizen R, Janssen PP, Wouters EF and Schols AM: Limb muscle dysfunction in COPD: Effects of muscle wasting and exercise training. Med Sci Sports Exerc 37: 2-9, 2005.

34. Teopompi E, Tzani P, Aiello M, Ramponi S, Andrani F, Marangio E, Clini E and Chetta A: Fat-free mass depletion is associated with poor exercise capacity irrespective of dynamic hyperinflation in COPD patients. Respir Care 59: 718-725, 2014.

This work is licensed under a Creative Commons Attribution-NonCommercial-NoDerivatives 4.0 International (CC BY-NC-ND 4.0) License. 